\title{
Cause-Specific School Absenteeism Monitoring Identifies Community Influenza Outbreaks
}

\author{
Jonathan Temte ${ }^{\star 1}$, Shari Barlow ${ }^{1}$, yenlik Zheteyeva ${ }^{2}$, Maureen Landsverk ${ }^{1}$, \\ Emily Temte', Amber Schemmel', Brad Maerz', Ron Gangon', Erik Reisdorf', \\ Pete Shult ${ }^{1}$, Mary Wedig ${ }^{1}$, Thomas Haupt ${ }^{3}$, Ashley Fowlkes ${ }^{2}$ and Amra Uzicanin ${ }^{2}$
}

${ }^{1}$ Family Medicine and Community Health, University of Wisconsin, Madison, WI, USA; ${ }^{2}$ Centers for Disease Control and Prevention, Atlanta, GA, USA; ${ }^{3}$ Wisconsin Department of Public Health, Madison, WI, USA

\section{Objective}

The Oregon Child Absenteeism due to Respiratory Disease Study (ORCHARDS) was implemented to assess the relationships between cause-specific absenteeism within a school district and medically attended influenza visits within the same community.

\section{Introduction}

Transmission and amplification of influenza within schools has been purported as a driving mechanism for subsequent outbreaks in surrounding communities. However, the number of studies assessing the utility of monitoring school absenteeism as an indicator of influenza in the community is limited. ORCHARDS was initiated to evaluate the relationships between all-cause (a-Tot), illness-related (aI), and influenza-like illness (ILI)-related absenteeism (a-ILI) within a school district and medically attended influenza A or B visits within the same community.

\section{Methods}

ORCHARDS was based at the Oregon School District (OSD), which enrolls 3,640 students at six schools in south-central Wisconsin. Parents reported influenza-like symptoms on an existing phone-based absenteeism reporting system. Attendance staff identified ILI using a simple case definition. Absenteeism was logged into the OSD's existing electronic information system (Infinite Campus), and an automated process extracted counts of a-Tot, a-I, and a-ILI each school day from 9/02/14 through 6/08/17.

Parents of students with acute respiratory infections (ARI) were invited to contact study staff who assessed the students' eligibility for the study based on presence of ILI symptoms. From 1/05/15 through $6 / 08 / 17$, data and nasal swabs were collected from eligible OSD students whose parents volunteered to have a study home visit within 7 days of ILI onset. Specimens were tested for influenza A and B at the Wisconsin State Laboratory of Hygiene using the CDC Human Influenza Virus Real-time RT-PCR Diagnostic Panel.

For community influenza, we used data from the Wisconsin Influenza Incidence Surveillance Project (WIISP) that monitors medically attended influenza using RT-PCR at five primary care clinics surrounding the OSD.

Data analysis: Over-dispersed Poisson generalized additive loglinear regression models were fit to the daily number of medically attended influenza cases and daily absenteeism counts from three sources (a-Tot, a-I, and a-ILI) with year and season (calendar day within year) as smooth functions (thin plate regression splines). Two subgroups of a-ILI representing kindergarten through 4th grade (K-4) and 5th-12th grade (5-12) were also evaluated.

\section{Results}

During the study period, 168,859 total absentee days $(8.57 \%$ of student days), 36,104 illness days (1.83\%), and 4,232 ILI days $(0.21 \%)$ were recorded. Home visits were completed on 700 children [mean age $=10.0 \pm 3.5(\mathrm{sd})$ years]. Influenza RT-PCR results were available for 695 (99.3\%) children: influenza A was identified in 54 $(13.3 \%)$ and influenza B in $51(12.6 \%)$ specimens. There were one large and early outbreak of influenza A (H3N2) followed by B in
2014/15, an extremely late combined outbreak of influenza A (H1N1) and $B$ in $2015 / 16$, and a combined outbreak of influenza A/(H3N2) and B in 2016/17. PCR detection of influenza A or B, as compared to no influenza, was strongly associated with a child with a-ILI-positive status (OR=4.74; 95\% CI: 2.78-8.18; $\mathrm{P}<0.001)$.

Nearly 2,400 medically attended ARI visits were reported during the study period. Of these, 514 patients were positive for influenza (21.5\%): 371 (15.5\%) influenza A and $143(6.0 \%)$ influenza B. The temporal patterns of medically attended influenza were very similar to influenza cases in OSD students.

Comparisons of the regression models demonstrated the highest correlation between absenteeism and medically attended influenza for $5^{\text {th }}-12^{\text {th }}$ grade students absent with ILI with a -1 day time lag and for all students with a-ILI with a -1 day lag (Table); a-I also had moderate correlation with a -15 day lag period.

\section{Conclusions}

Cause-specific absenteeism measures (a-I and a-ILI) are moderately correlated with medically attended influenza in the community and are better predictors than all-cause absenteeism. In addition, a-I preceded community influenza cases by 15 days. The monitoring system was easily implemented: a-I surveillance was fully automated and a-ILI required only minor review by attendance staff. The resulting correlations were likely lowered by the presence of other viruses that resulted in a-ILI (e.g., adenovirus) and by breaks in the school year during which absenteeism data did not accrue.

Automated systems that report cause-specific absenteeism data may provide a reliable method for the early identification of influenza outbreaks in communities. From a preparedness perspective, 15-day advance warning is significant. The addition of a laboratory component could increase usefulness of the cause-specific student absenteeism monitoring as an early-warning system during influenza pandemics.

Correlation and lag time comparing absenteeism to medically attended influenza

\begin{tabular}{|c|c|c|c|}
\hline Absenteeism type & Correlation & Lag (in days) & P-value \\
\hline a-Tot & 0.278 & +9 days & $<0.001$ \\
\hline a-I & 0.472 & -15 days & $<0.001$ \\
\hline a-ILI & 0.480 & -1 day & $<0.001$ \\
\hline a-ILI (K-4) & 0.298 & -1 day & $<0.001$ \\
\hline a-ILI (5-12) & 0.497 & -1 day & $<0.001$ \\
\hline
\end{tabular}

\section{Keywords}

school; absenteeism; influenza

\section{Acknowledgments}

This study was possible thanks to a collaboration between the Centers for Disease Control and Prevention, Wisconsin State Lab of Hygiene, Wisconsin Department of Health Services, University of Wisconsin Department of Family Medicine and Community Health, Quidel Corporation, and Oregon School District staff, students, and families

\section{*Jonathan Temte}

E-mail: jon.temte@fammed.wisc.edu 